\title{
Tunable Optical Vortices Generated by Self-Assembled Defect Structures in Nematics
}

\author{
Péter Salamon, ${ }^{1,2, *}$ Nándor Éber, ${ }^{1}$ Yuji Sasaki, ${ }^{3}$ Hiroshi Orihara, ${ }^{3}$ Ágnes Buka, ${ }^{1}$ and Fumito Araoka ${ }^{2, \dagger}$ \\ ${ }^{1}$ Institute for Solid State Physics and Optics, Wigner Research Centre for Physics, Hungarian Academy of \\ Sciences, Budapest, P.O. Box 49, 1525, Hungary \\ ${ }^{2}$ RIKEN Center for Emergent Matter Science, 2-1 Hirosawa, Wako, Saitama 351-0198, Japan \\ ${ }^{3}$ Division of Applied Physics, Faculty of Engineering, Hokkaido University, North 13 West 8, Kita-ku, Sapporo, \\ Hokkaido 060-8628, Japan
}

(Received 26 April 2018; revised manuscript received 25 July 2018; published 3 October 2018; corrected 11 October 2018)

\begin{abstract}
A stable lattice of topological defects occurring at the electric reorientation of a nematic fluid is studied by quantitative polarimetry and laser diffraction. Generation of optical vortices by topological defects is demonstrated in the case of two distinct mechanisms. First, individual defects convert circularly polarized light partially into a vortex beam with opposite handedness, while beams diffracted on the defect lattice do not carry vorticity. Second, dislocation of the lattice structure is a topological defect on a larger length scale; then beams diffracted on a single dislocation possess optical vortex character. The vortex-generation efficiency is tunable by the applied voltage.
\end{abstract}

DOI: 10.1103/PhysRevApplied.10.044008

\section{INTRODUCTION}

Topological defects are universal in nature. They are present when a discontinuity in an order-parameter field of a system cannot be removed by continuous transformations. In optics, topological defects appear in phase singularities of light carried by optical vortices [1]. In the singularity, the electric field of light is zero, and the spatial distribution of the phase resembles the structure of a screw dislocation. Therefore, the optical vortex exhibits a helical phase front, (i.e., the phase propagates along a helix). One spectacular property of optical vortices is that they carry orbital angular momentum, $l \hbar$ per photon, where $l$ is the topological charge of the vortex. For cylindrically symmetric laser beams of finite diameter, the complex amplitude of light can be given as a superposition of $u_{p l}(r, \phi, z)$ Laguerre-Gaussian modes [1], where $r$, $\phi$, and $z$ are the usual notation for cylindrical coordinates: radius, azimuth angle, and propagation direction, respectively. With $p=l=0$, we get a common Gaussian beam, while $p=0, l=1$ represents the simplest vortex beam. Such a vortex beam is easily recognized by its doughnutshaped intensity profile. Its topological charge $l$ is usually determined by observation of its interference pattern with a Gaussian reference beam; $l$ equals the number of spiral arms seen in the interferograms [1].

Besides pure scientific interest, optical vortices have gained applications on a wide spectrum [2]. They are used

\footnotetext{
*salapeter@gmail.com

†fumito.araoka@riken.jp
}

in superresolution microscopy $[3,4]$ as well as in laser tweezers to precisely manipulate particles on a microscopic scale [5,6]. It was demonstrated that catching and transportation of particles in air is possible by the use of optical vortices $[7,8]$. Because of the stability of the vortex mode, they have great potential in optical communication technologies [9]. Furthermore, the use of highperformance vortex retarders in stellar coronagraphy has resulted in significant advances in astronomical imaging [10-12]. Therefore, extensive research is devoted to find various modes for generation of optical vortices. One of the most-commonly-applied methods is the use of a fork grating (or a holographic pattern) that creates an optical vortex in the first-order diffracted laser beam [1,5,13]. A phase mask of spiral phase patterns $[6,14]$ or a phase plate of birefringent solid polymer structures with cylindrical symmetry [15] is a convenient tool and has been commercialized in recent years. However, most of these methods or elements use solid structures and thus are basically usable in fixed optical systems designed for specific wavelengths. With the help of computer-controlled spatial light modulators, both holographic and phase-mask patterns can be realized. However, the finite resolution of such pixelated devices limits the quality of the vortex beams generated.

In liquid crystals (LCs), being highly birefringent optical materials, the local optical axis, the director, can be affected and controlled by external electric or magnetic fields, as well as by boundary conditions [16]. Furthermore, LCs are capable of self-assembly due to their orientational elasticity; they can maintain topological defect 
structures, which gives them the potential for being smart materials for applications quite different from their typical use in display technologies. For example, LCs can be used as tunable diffraction gratings [17-19] based on nematic-defect-mediated colloidal crystals [20] or on flexoelectric domains [21]. Their unique optical properties created by self-assembly makes LCs promising materials also in information technologies $[22,23]$. Their polymerized structures offer extraordinary mechanical properties [24-26], and their self-assembled patterns are also suitable for optical vortex generation, without suffering from the adverse effect of low resolution.

With use of liquid crystals, circular phase plates have been created by special boundary conditions imposed by circular rubbing [27,28], photoalignment [29-37], or micropatterned structures [38], and the resulting structures have been used to generate optical vortices. Arrays of defects generated by standing waves [39] or by selfassembling smectic focal conic domains [40] were also observed and used to create optical vortices. Such singular light beams have been generated by laser-written disclinations [41] or point-defect structures [42] in cholesteric liquid crystals. Dislocation textures in liquid crystals can be used as fork gratings to diffract laser beams; optical vorticity was confirmed in the first-order diffracted beams [41-46]. On the other hand, topological charges of single defects in nematics are also available for optical vortex generation by transfer of the topological singularity from the director structure to the light phase as demonstrated in nematic droplets [47] or in a sandwich cell [48]. The topological charge of the optical vortex generated is the double of that of the nematic defect $(l=2 s)$. By illumination of one defect by circularly polarized light, it was shown that the output beam partially becomes of vortex type with opposite handedness of the circular polarization, while the other part of the light leaves the sample unaffected. Notably in the latter work, tunability of liquid crystals by the electric field was used to adjust the optical vortex conversion efficiency from the randomly distributed defects of $s= \pm 1$ topological charge. To facilitate future applications, the position and topological charge of the defects should be kept under control. That could be achieved by the use of patterned electrodes [49-54], by doping a nematic with a nonmesogenic material [55], or by photopatterned substrates [56,57]. By the use of the nonlinear optical behavior of an initially homogeneous nematic layer, charge flipping of a vortex beam was also demonstrated [58].

Recently, it was reported that by use of a special perfluoropolymer surface-aligning layer with liquid crystals of high conductivity, it is possible to have an electrically switchable, tunable, self-assembled grid structure of topological defects [59]. Later, it was shown that the exact positions of the defect cores can be fixed, but without losing the switchability and tunability, by partial polymerization of the sample [60]. Here we report our results on how this electrically tunable defect pattern can be used in generating adjustable optical vortices. We study their creation on different levels: using the individual defects as vortex retarders, as well as using diffraction on dislocations in the defect lattice as sources of vortices.

\section{EXPERIMENTAL PROCEDURES}

We use a mixture of $4 \alpha, 4^{\prime} \alpha$-dialkyl- $1 \alpha, 1^{\prime} \alpha$ bicyclohexyl- $4 \beta$-carbonitrile (CCNmn) liquid crystals that exhibits a nematic phase at room temperature [59]. The 1:1 weight ratio mixture of $\mathrm{CCN} 47$ and CCN55 nematics is doped with $1 \mathrm{wt} \%$ tetrabutylammonium benzoate salt in the isotropic phase to increase its electrical conductivity and thus fulfill the requirement for the grid-pattern generation [59]. At $25^{\circ} \mathrm{C}$, at which all measurements are conducted, the mixture has a large negative dielectric anisotropy $\left(\varepsilon_{a} \approx-8\right)$ and a low birefringence $\left(n_{a} \approx 0.03\right)$. Sandwich cells made of glass plates covered with $0.5-5-\mathrm{mm}$-wide stripes of indium tin oxide transparent electrodes are filled with the doped LC. The stripes on the opposite plates are perpendicular to each other, forming a matrix structure of square and rectangular pixels. Mylar spacers are used to set the thickness $d$ of the cells. In the studies presented here, we use 21.4- and 26.3- $\mu \mathrm{m}$-thick cells. The inner surfaces of the cells are coated with approximately-120-nm-thick polymer layers prepared by spin coating a 3 -wt $\%$ solution of a perfluoropolymer (33 wt \% CYTOP CTX-809A, containing 9 wt $\%$ polymer, in CT-Solv 180 solvent, AGC Corp.) at $800 \mathrm{rpm}$ for $9 \mathrm{~s}$, then at $3000 \mathrm{rpm}$ for $15 \mathrm{~s}$. The freshly coated substrates are dried at $70{ }^{\circ} \mathrm{C}$ for 30 min, then at $120^{\circ} \mathrm{C}$ for more than $30 \mathrm{~min}$. At $25^{\circ} \mathrm{C}$, the CYTOP layer provides a homeotropic alignment condition [61], which means that the director is aligned perpendicularly at the surface. During all experiments presented here, the samples are kept in a heat stage (HCS402, Instec Inc.) at $25 \pm 0.1{ }^{\circ} \mathrm{C}$ to avoid uncertainties in measurements related to the change of material parameters (e.g., optical or dielectric anisotropy) due to temperature fluctuations. An ac electric voltage is applied from a digital function generator (AFG3021B, Tektronix Inc.) through a high-voltage amplifier (NF Corp.).

Measurements are performed with a multifunctional optical setup, which works either in an imaging mode or in a diffraction mode (see Fig. 1, where filters and irises are not shown for simplicity). In imaging mode (mirror M2 and beam splitter BS3 removed), white-light or monochromatic polarizing microscopy is possible. In addition, with the help of insertable wave plates, an imaging-polarimetry technique is implemented for quantification of the effective birefringence in the plane of the sample [62]. In diffraction mode, with condenser and imaging objectives removed, the diffraction pattern generated by the defect lattice in 


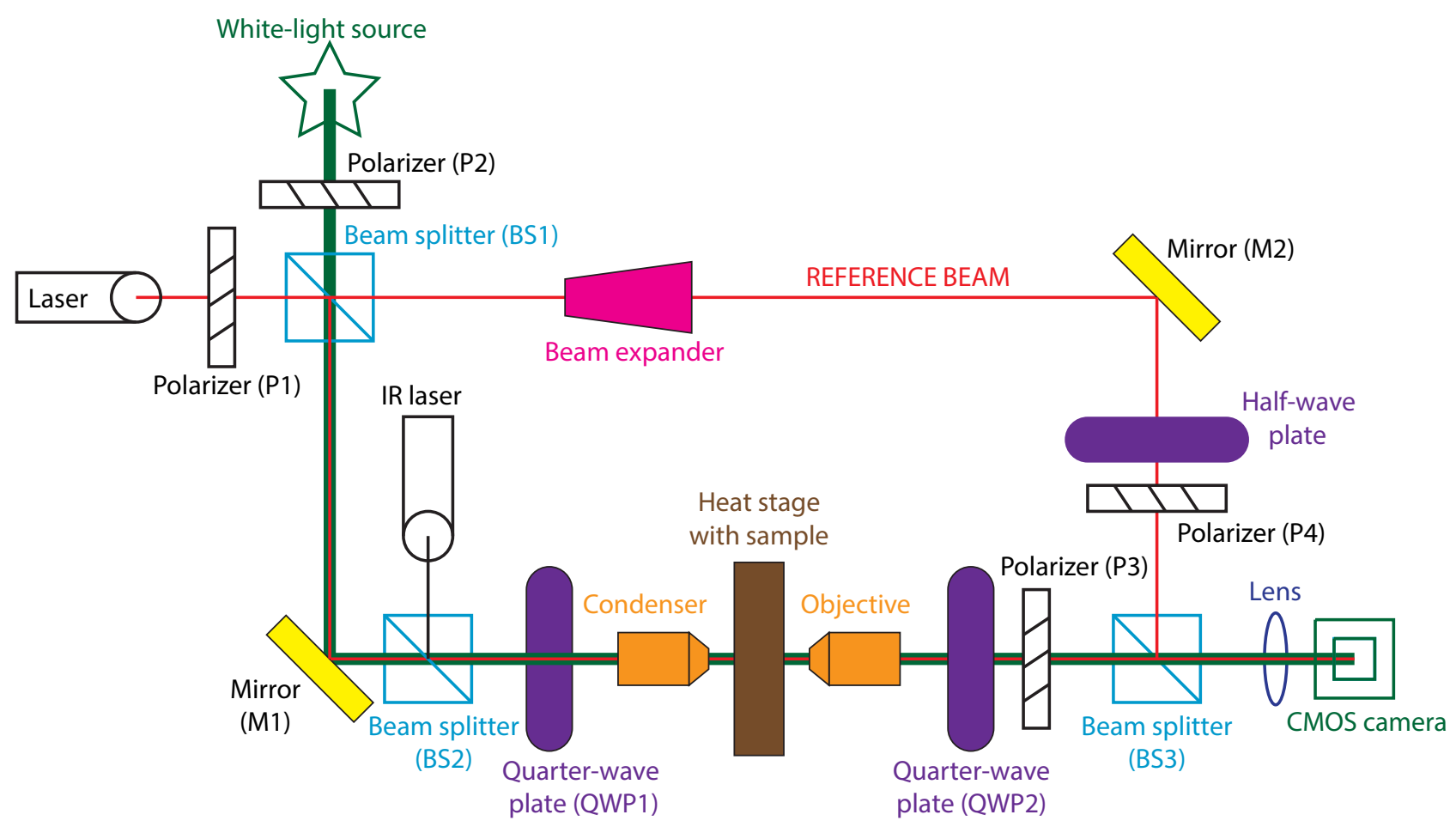

FIG. 1. The optical setup used as a polarizing microscope and an imaging polarimeter (mirror M2 removed), and as a Mach-Zehnder interferometer (mirror M2 included). For simplicity, filters and irises are not shown.

the sample can be recorded. The setup can be transformed to a Mach-Zehnder interferometer usable in both imaging mode and diffraction mode. For measurement of interference, the size and the intensity of the reference beam are adjusted by a beam expander and a half-wave plate, respectively. The interferometry allows us to confirm the presence of optical vortex components and characterize their topological charge. Measurements are done with whitelight illumination, and with use of monochromatic light from three different lasers: a red He-Ne laser $(\lambda=633$ $\mathrm{nm})$, a green diode-pumped solid-state laser $(\lambda=532 \mathrm{~nm})$, and a blue diode laser $(\lambda=450 \mathrm{~nm})$. For polarimetry, white light is used with a bandpass filter with 633-nm peak wavelength with $10-\mathrm{nm}$ full width at half maximum. The microscopic, diffraction, and interference images are recorded by an 8-bit digital CMOS camera (PL-B762, Pixelink) with $752 \times 480$ pixels. Additionally, an infrared (IR) diode-pumped solid-state laser $(\lambda=1064 \mathrm{~nm})$ can be focused onto the sample, allowing optical manipulation of the defects via local heating. Beam splitter BS2 is included in the setup only when the manipulation of the defect structure is performed by the IR laser.

\section{RESULTS}

\section{A. Optical characterization: imaging polarimetry and diffractometry}

In homeotropic samples, where the director is considered fixed at the substrates in the direction of the surface normals, using compounds with $\varepsilon_{a}<0$, one can induce a Freedericksz transition with an electric field parallel to the initially homogeneous bulk homeotropic alignment. In such geometry, the energetically favorable director reorientation, when the director tends to be perpendicular to the electric field, is azimuthally degenerate. As a consequence, the so-called umbilical defects appear, which are not strictly singularities in the director field; their cores are not localized point defects, but are places where the director escapes to the original homeotropic direction, out of the cell plane [16,63-65].

There is a specific voltage and frequency range where formation of a lattice of umbilical defects has a lower threshold voltage $U_{\text {th }}$ than that of the homogeneous Freedericksz transition or that of the appearance of randomly scattered umbilical defects [59]. Such defect-lattice formation is considered as a result of an instability related to the large difference between the conductivities of the LC and the CYTOP layer, leading to internal voltage attenuation. By application of voltage to the overlapping electrodes, a lattice of umbilical defects can be generated [Figs. 2(a) and 2(b); the sample thickness is $d=26.3 \mu \mathrm{m}]$. As the periodicity of the lattice is much smaller than the electrode width, the electrode edges have no direct influence on the position of the defects, nor on their periodicity, although they force the lattice direction to be parallel with them. The lattice size depends on the combination of various material constants and on the parameters of the driving voltage ( $U$ and $f$ ), and importantly, it directly scales with 

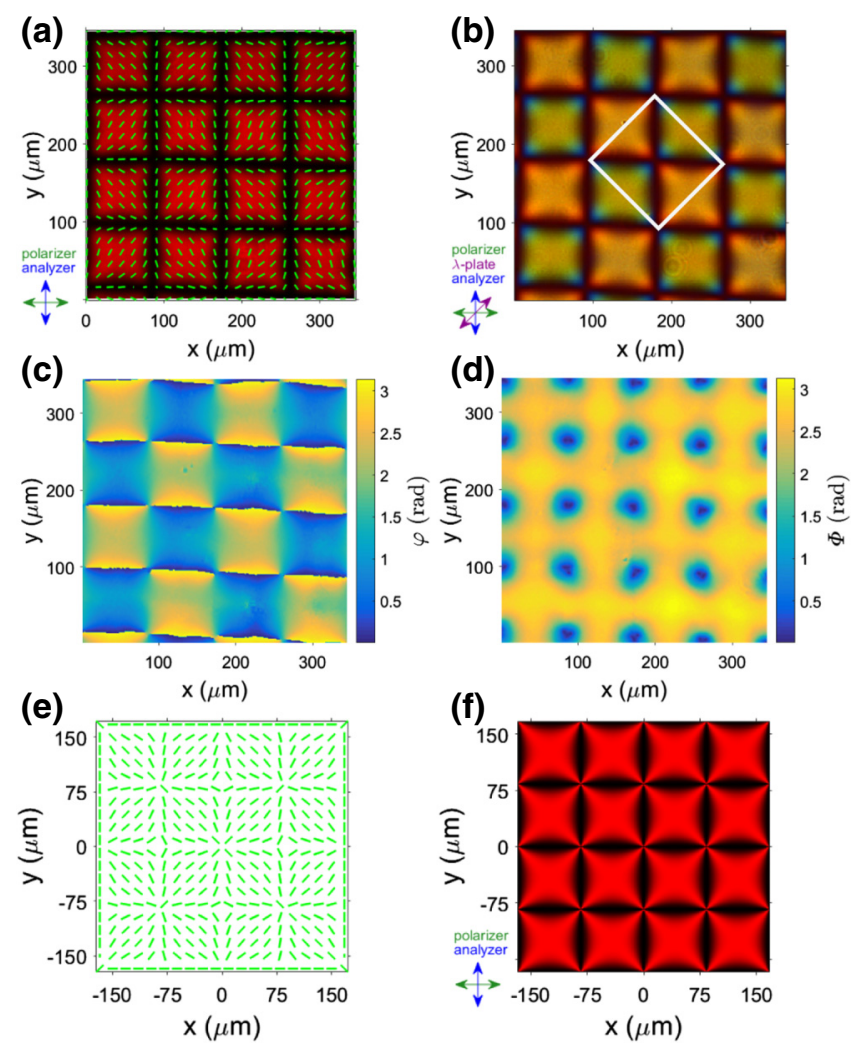

FIG. 2. Snapshots of polarization micrographs under crossed polarizers (a) without and (b) with a $\lambda$ plate inserted. In (b), the white square represents a possible choice of the primitive cell of the defect lattice. (c) Color map of the azimuthal angle $\varphi(x, y)$ of the effective slow axis and (d) the magnitude of the retardation as the effective phase shift $\Phi(x, y)$. The experimental conditions are $f=22 \mathrm{~Hz}, U=1.07 U_{\mathrm{th}}$, and $d=26.3 \mu \mathrm{m}$. Green rods in (a) represent visualization of the average in-plane director $\tilde{\mathbf{n}}$. (e) The simplified two-dimensional director field described by Eqs. (7) and (8). (f) Simulated texture of the two-dimensional director field with $\Phi=2.8$ at $633-\mathrm{nm}$ wavelength and with crossed polarizers.

the cell thickness [59]. The optical properties of the defect lattice are investigated by two complementary techniques: polarimetry and diffraction.

Using the imaging-polarimetry technique, we quantitatively map the effective birefringence in the plane of the sample. In general, the director is a pseudovector field: $\mathbf{n}(x, y, z)=\left(n_{x}(x, y, z), n_{y}(x, y, z), n_{z}(x, y, z)\right)$, with $|\mathbf{n}|=1$. With this polarimetric technique, we can determine only an average director in the $x-y$ (sample) plane. Figure 2(c) depicts the color map of the azimuthal angle $\varphi(x, y)$ of the effective slow axis (which is actually the projection of the average director), and Fig. 2(d) shows the color map of the magnitude of the effective retardation (phase shift)

$$
\Phi(x, y)=\frac{2 \pi}{\lambda} \int_{-d / 2}^{d / 2}\left(n(z)-n_{o}\right) d z
$$

with

$$
n(z)=\frac{n_{e} n_{o}}{\sqrt{n_{o}^{2}\left(n_{x}^{2}+n_{y}^{2}\right)+n_{e}^{2} n_{z}^{2}}},
$$

where $n_{o}$ and $n_{e}$ are the ordinary and extraordinary refractive indices, respectively, $n(z)$ is the effective local index corresponding to the out-of-the-sample-plane tilt of the director, and $d$ is the cell thickness. For each pixel, the average director $\tilde{\mathbf{n}}=\left(\tilde{n}_{x}, \tilde{n}_{y}, \tilde{n}_{z}\right)$ can be calculated such that

$$
\Phi(x, y)=\frac{2 \pi d n_{o}}{\lambda}\left[\frac{n_{e}}{\sqrt{n_{o}^{2}\left(\tilde{n}_{x}^{2}+\tilde{n}_{y}^{2}\right)+n_{e}^{2} \tilde{n}_{z}^{2}}}-1\right] .
$$

The projection of the average nematic director field on the sample plane determined experimentally is plotted as green rods on top of the polarizing micrograph taken with crossed polarizers and monochromatic red light in Fig. 2(a). Shorter rods mean smaller director tilt from the plane normal and thus correspond to lower birefringence. Furthermore, Fig. 2(b) presents a snapshot taken with an additional $\lambda$ plate (full-wave plate) inserted (with white-light illumination). It is evident from the figures that defects with $s=-1$ and $s=+1$ topological charges alternate in the lattice with a distance $D$ between them. Consequently, the primitive cell of the lattice is a centered square positioned at $45^{\circ}$ with a lattice constant of $\sqrt{2} D$. A possible choice of the primitive cell of the lattice is shown as a white box in Fig. 2(b), with the $s=-1$ defects at the corners and the $s=+1$ defect in the center.

To clearly understand the experimental findings, we perform optical calculations based on the continuum theoretical description [59] of the system considered. The three-dimensional director field of the defect lattice in the linear approximation of the director (assuming $n_{x} \ll 1$ and $\left.n_{y} \ll 1\right)$ is given by

$$
\begin{gathered}
n_{x}(x, y, z)=-\theta(z) \sin (\sqrt{2} q x), \\
n_{y}(x, y, z)=\theta(z) \sin (\sqrt{2} q y), \\
n_{z}(x, y)=\sqrt{1-n_{x}^{2}-n_{y}^{2}},
\end{gathered}
$$

where $\theta$ is the tilt angle of the director with respect to the sample normal and $q$ is the characteristic wave number of the pattern. This director field, which is actually a $45^{\circ}$-rotated version of that used in Ref. [59], holds near the threshold voltage $U_{\text {th }}$, where the distortion is still small $(\theta \ll 1)$, and describes defects with alternating $s=+1$ and $s=-1$ topological charges, which are positioned along lines parallel to the $x$ and $y$ axes. The distance between neighboring defects is $\Lambda=\sqrt{2} \pi / q$. On increase of the voltage, the distortion is enhanced and director gradients 

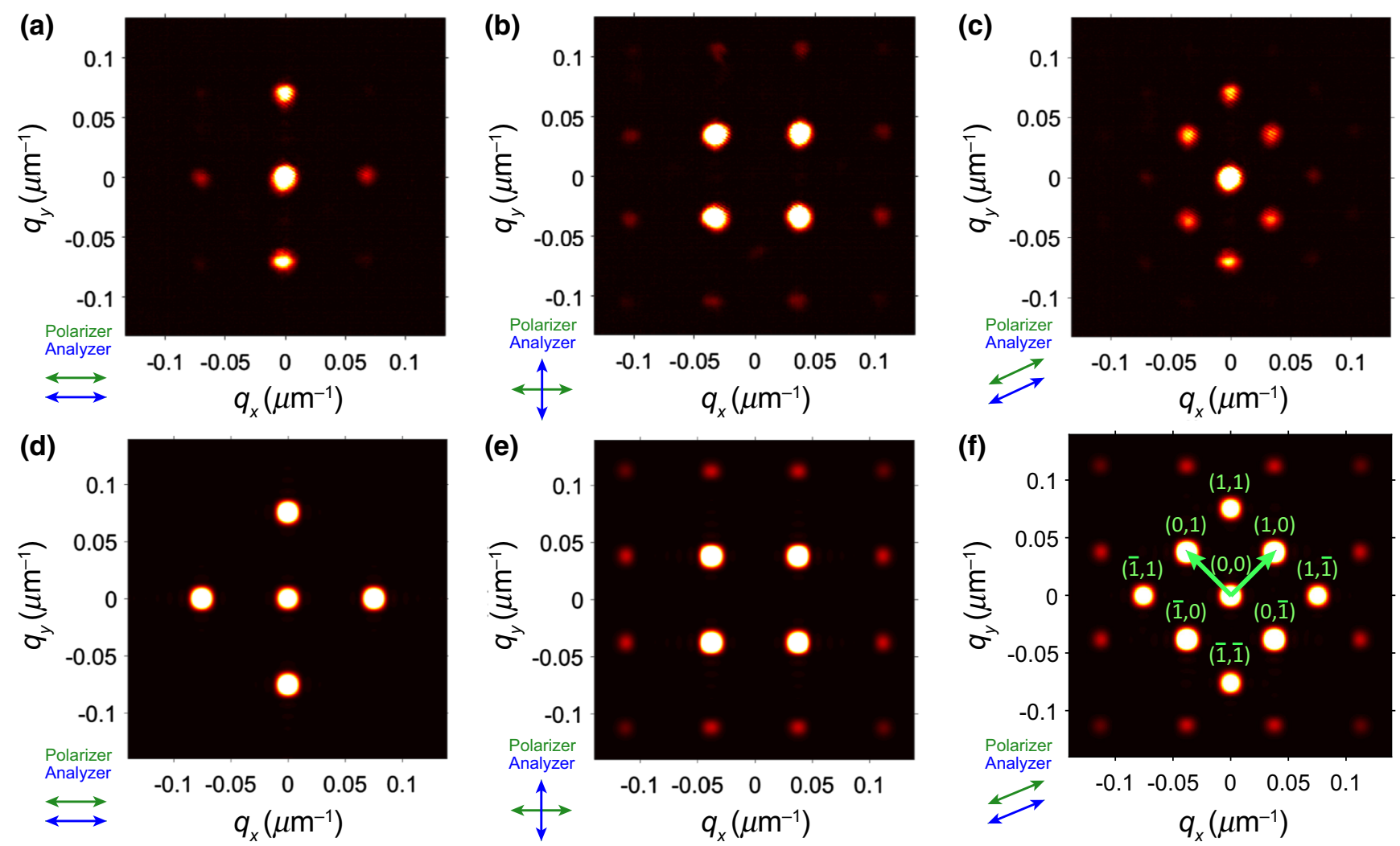

FIG. 3. Experimental (a)-(c) and simulated (d)-(f) diffraction patterns with several different polarizer settings. The assigned base vectors in Fourier space and the indexing of spots are indicated in (f).

are mostly restricted to a narrow boundary layer near the substrate and to narrow regions around the defect cores. In the very-high-voltage limit $\left(U \gg U_{\text {th }}\right)$ one thus arrives at a simplified two-dimensional (2D) director field of

$$
\begin{aligned}
& n_{x}(x, y)=-\frac{\sin (\sqrt{2} q x)}{\sqrt{\sin ^{2}(\sqrt{2} q x)+\sin ^{2}(\sqrt{2} q y)}}, \\
& n_{y}(x, y)=\frac{\sin (\sqrt{2} q y)}{\sqrt{\sin ^{2}(\sqrt{2} q x)+\sin ^{2}(\sqrt{2} q y)}} .
\end{aligned}
$$

This 2D director structure shown in Fig. 2(e) has the same azimuthal angles as the three-dimensional distortion given in Eqs. (4)-(6), but is assumed to be independent of $z$. It has a constant retardation $\Phi$ everywhere but at the singular defect cores. This director field, which approximates the actual director structure of the experimental defect lattice at high voltages, is used as the starting point for the optical calculations. The 2D approximation is expected to give a good description of the grid pattern when the size of the defect cores is significantly smaller than the cell thickness (the lattice size of the grid structure is proportional to the thickness). In the case of a single umbilical defect, the core size $\zeta$ depends on the applied voltage relative to the threshold: $\zeta \sim(d / \pi)\left[\left(U / U_{\text {th }}\right)^{2}-1\right]^{-1 / 2}[63]$. This expression may not be precise for the grid pattern; nevertheless, its application is the only way to give a crude estimate of the validity limit for the $2 \mathrm{D}$ approximation. If the core is regarded as small at $\zeta / d<0.1$, then $U>3.3 U_{\text {th }}$ should stand for the validity range of the $2 \mathrm{D}$ approximation.

By knowing the director field, we can calculate the electric field vectors $\mathbf{E}_{o}(x, y)$ and $\mathbf{E}_{e}(x, y)$ (subscripts $o$ and $e$ represent the ordinary and extraordinary light components), which determine the intensity and the polarization of the light exiting the sample, using a Jonesmatrix method $[66,67]$. Our calculations consider the simplest case of parallel input rays, which is shown to give qualitatively-very-comparable results for experiments and simulations performed even with numerical apertures of 0.4 [67]. Additionally, effects such as ray deflection and focusing-defocusing can be ignored in our case due to the exceptionally low refractive-index anisotropy of the LC mixture used.

An example of a calculated polarizing-microscope image can be seen in Fig. 2(f), for which approximately the same parameters $(\Phi=2.8$, red light of $633-\mathrm{nm}$ wavelength, and same defect distances) are used as for the experimental data shown in Fig. 2. The Jones-matrix 
calculation reconstructs qualitatively well the measured image with crossed polarizers; some differences can be found in the vicinity of the defect cores, which is because in the case of the experiment, the phase difference decreases there, in contrast to the simplified 2D model, where $\Phi$ is constant, except precisely at the defect cores.

As clarified by the polarimetry result, the defect lattice in the present study is essentially the $2 \mathrm{D}$ periodic structure of the nematic director field. Therefore, it works as a phase grating. By wide-area illumination, clear far-field diffraction patterns are observed, depending on the light polarization states [Figs. 3(a)-3(c)]. For better visibility, the color scale is different for all plots in Fig. 3. Knowing the electric field vectors $\mathbf{E}_{o}(x, y)$ and $\mathbf{E}_{e}(x, y)$ of the light exiting from the sample, we can simulate the patterns. The field vectors $\mathbf{E}_{o}^{d}\left(q_{x}, q_{y}\right)$ and $\mathbf{E}_{e}^{d}\left(q_{x}, q_{y}\right)$ of the diffracted light correspond to the 2D Fourier transforms of the field vectors at the exit from the sample:

$$
\begin{aligned}
& \mathbf{E}_{j}^{d}\left(q_{x}, q_{y}\right) \propto \iint \mathbf{E}_{j}(x, y) \exp \left(-i q_{x} x-i q_{y} y\right) d x d y \\
& \quad j \rightarrow o, e .
\end{aligned}
$$

Figures 3(d)-3(f) show the computed diffraction pattern obtained with the two-dimensional director distribution under the assumption of the normal incidence of light. As the unit cell of the defect lattice is at $45^{\circ}$ to the $x$ and $y$ axes, the reciprocal lattice is similarly rotated by $45^{\circ}$. The relative intensities and thus the visibility of the diffraction spots depend on the polarization states of light: the spot intensities depend on the angle $\beta$, which the polarizer encloses with the $x$ axis. The most-intense diffraction spots are indexed in Fig. 3(f) following the Miller convention. For parallel and crossed polarizers, the absolute diffraction efficiencies as a function of $\beta$ for different groups of peaks can be seen in Figs. 4 and 5, respectively. It is worth noting that while the peak positions shown by the experiments and the simple 2D model match well, there are mismatches in the $\beta$ dependence of spot intensities, and the relative intensities in some cases. The physical reason for this will be discussed elsewhere.

The efficiency of light diffraction on the grid pattern depends on the director profile as well as on the polarizer settings. For example, the central $(0,0)$ spot is extinct at crossed polarizers, and other spot intensities depend on the angle $\beta$, which the polarizer encloses with the $x$ axis.

The diffraction efficiencies for several spots are calculated in the case of various polarizer-analyzer settings by use of the $2 \mathrm{D}$ approximation of the director structure. Inspection of the simulation results shows that due to the symmetry of the defect lattice, the diffraction spots [indexed in Fig. 3(f)] can be separated into three groups: the central $(0,0)$ spot, the group-A $(1,0),(0,1),(\overline{1}, 0)$, and $(0, \overline{1})$ spots, and the group-B $(1,1),(1, \overline{1}),(\overline{1}, 1)$, and $(\overline{1}, \overline{1})$ spots. The behavior of spots belonging to the same group
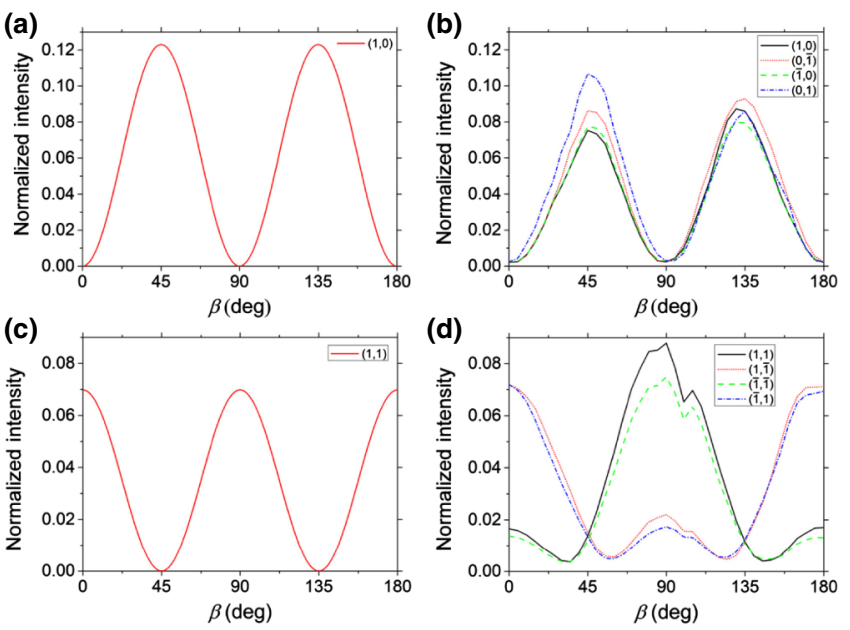

FIG. 4. Comparison of the theoretically expected and experimentally obtained dependence of selected spot intensities on the polarizer angle $\beta$ for a parallel polarizer and analyzer. (a) Calculated values for the $(1,0)$ spot, (b) measured values for the spots of group A, (c) calculated values for the $(1,1)$ spot, and (d) measured values for the spots of group B.

is identical but differs from that of the members of the other groups. Figures 4(a) and 4(c) depict the calculated angular $\beta$ dependence of the normalized intensity of the representative $(1,0)$ and $(1,1)$ spots, $I_{1,0}(\beta)$ and $I_{1,1}(\beta)$, respectively, at a parallel polarizer and analyzer setting; Figs. 5(a) and 5(c) depict the same, but at a crossed polarizer and analyzer setting. Normalization is done with respect to the total incoming intensity.

To allow a detailed comparison of experimental results and theoretical expectations, the measured angular
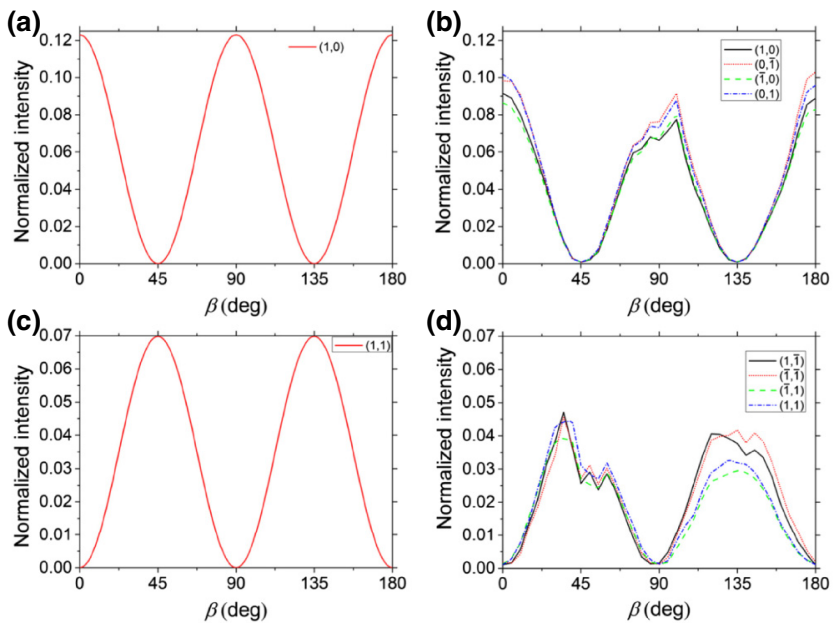

FIG. 5. Comparison of the theoretically expected and experimentally obtained dependence of selected spot intensities on the polarizer angle $\beta$ for a crossed polarizer and analyzer. (a) Calculated values for the $(1,0)$ spot;,(b) measured values for the spots of group A, (c) calculated values for the $(1,1)$ spot, and (d) measured values for the spots of group B. 


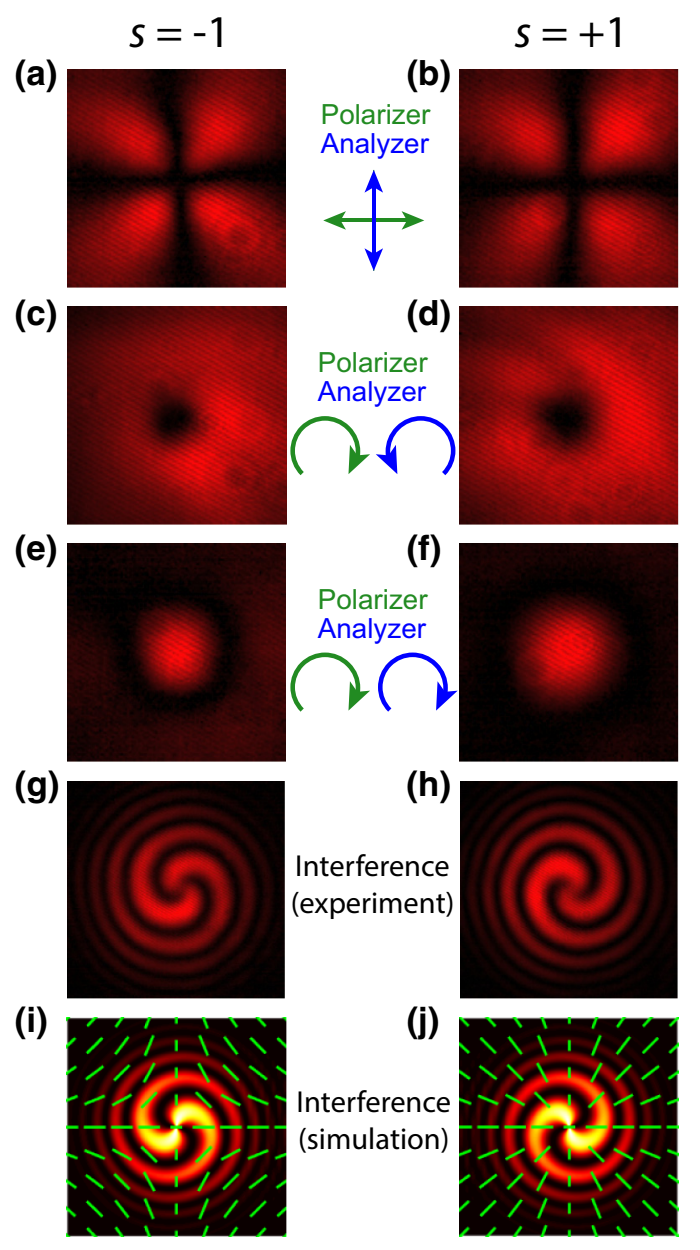

FIG. 6. (a)-(f) Snapshots $\left(35 \times 35 \mu \mathrm{m}^{2}\right)$ of the neighborhood of selected $s= \pm 1$ defects: (a),(b) for crossed polarizers, (c),(d) between a left-circular and a right-circular polarizer, and (e),(f) between two left-circular polarizers. (g),(h) Corresponding interferograms of the right-handed circularly polarized component obtained with a polarized Gaussian reference beam, whose swirling phase patterns prove the vortex-beam generation. The experimental conditions are $f=60 \mathrm{~Hz}, U=12 \mathrm{~V} \mathrm{rms}$, and $d=21.4 \mu \mathrm{m}$. (i),(j) Calculated spiral interferograms for the 2D deformation, showing good agreement with experimental ones.

dependence of the normalized intensities of selected spots is plotted in Figs. 4(b) and 4(d) for the parallel polarizer and analyzer setting and in Figs. 5(b) and 5(d) for the crossed polarizer and analyzer setting. The intensity $I_{i j}$ of the diffraction spot with index $(i, j)$ is calculated by summing the pixel intensities within a square area enclosing the named spot. For the normalization, the total intensity is calculated by summing the intensities of the nine indexed spots at parallel and crossed polarizer and analyzer settings. The intensities of higher-order diffractions (not indexed) are substantially weaker, so they are ignored.

Comparing the theoretical and experimental results in Figs. 4 and 5, one can immediately notice a typically good qualitative agreement in the shape of the curves.
The minima and maxima are at the expected angular positions; the maximum intensities are in the expected order. [The sudden deviations from the sinusoidal shape seen in Figs. 5(b) and 5(d) are attributed to unexpected temporary fluctuations of the illuminating-laser intensity during the measurement.] The only significant difference is detectable in Fig. 4(d), which shows a symmetry breaking in the angular dependence of the intensities of the spots of group B; namely, the behavior of the $(1,1)$ and $(\overline{1}, \overline{1})$ spots differs from that of the $(1, \overline{1})$ and $(\overline{1}, 1)$ spots. The physical reason for this symmetry breaking will be discussed elsewhere in detail; the origin of this effect is expected to be the cumulative consequence of factors not taken into account in the simulations. These may include a small deviation from the normal incidence of light, a small gradient of the sample thickness, a deviation from the cylindrical cross section

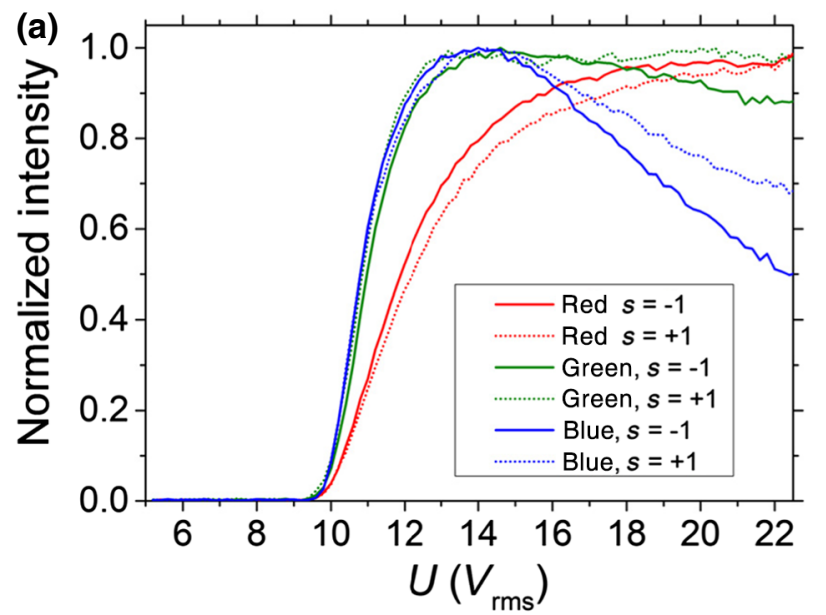

(b)

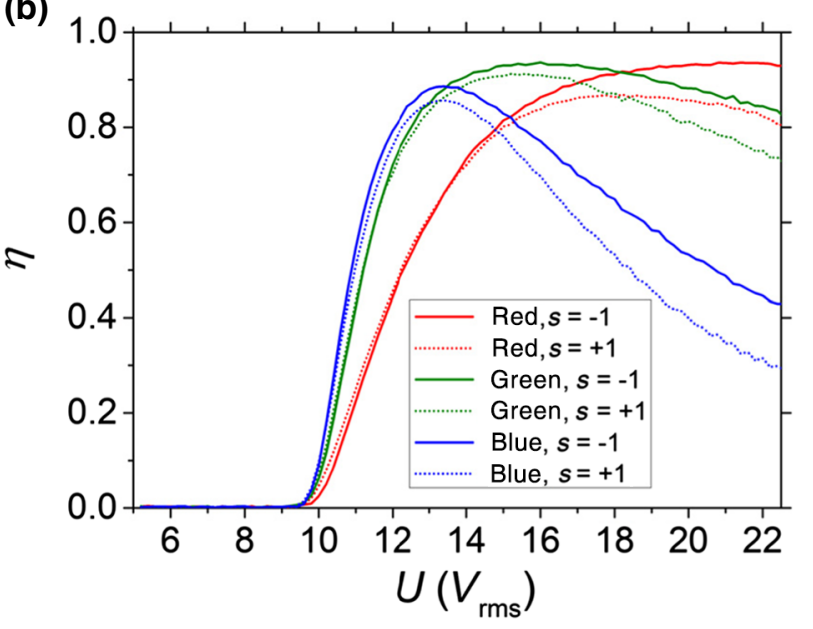

FIG. 7. (a) Voltage dependence of the transmitted intensity of laser beams integrated over a square area around defects with topological charge of $s=-1$ and $s=+1$. (b) Voltage dependence of the vortex-generation efficiency $\eta$ for defects with topological charge of $s=-1$ and $s=+1$. The red, green, and blue curves correspond to illumination with laser light of the corresponding color. 

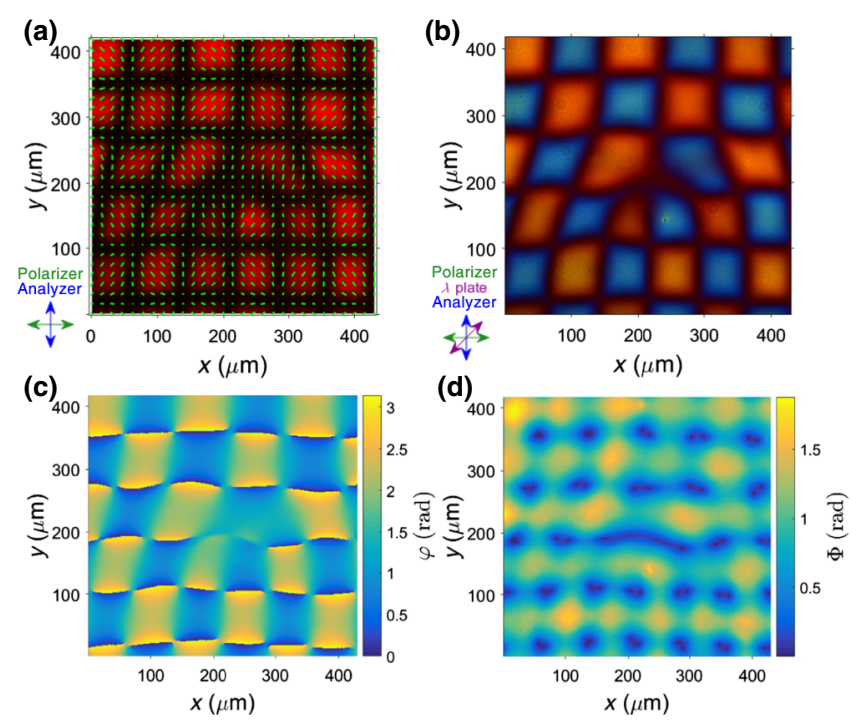

FIG. 8. Snapshots of polarization micrographs of a dislocation in the grid pattern under crossed polarizers (a) without and (b) with a $\lambda$ plate inserted. (c) Color map of the azimuthal angle $\varphi(x, y)$ of the effective slow axis and (d) the magnitude of the retardation as the effective phase shift $\Phi(x, y)$. The experimental conditions are $f=22 \mathrm{~Hz}, U=1.05 U_{\text {th }}$, and $d=26.3 \mu \mathrm{m}$. Green rods in (a) represent visualization of the average in-plane director $\tilde{\mathbf{n}}$.

of the illuminating Gaussian beam, a difference between the centers of the Gaussian beam and the defect lattice, and the curvature of the light path through the nematic LC layer (shadowgraph effects). This assumption is reinforced by the fact that calculation of the diffracted image even from the experimentally determined polarimetric data does not reproduce the symmetry breaking observed in Fig. 4(d).

\section{B. Vortex-beam generation from single defect points}

Figures 6(a) and 6(b) show polarizing micrographs of the $s= \pm 1$ topological defects taken with the imaging mode with crossed polarizers and limited-area laser-light illumination. The transmission intensities in these single defect areas are measured as a function of the applied voltage $U$, presented in Fig. 7(a). The intensity values are obtained by integration over the images of the selected square area corresponding to Fig. 6. The transmission intensity $I_{T, \text { cross }}(U)$ drastically changes at a certain threshold voltage, according to the sharp transition from the homeotropic state to the defect-lattice state. This behavior resembles that of the conventional Freedericksz transition, but occurs at a considerably higher threshold voltage compared with the usual sample cells with alignment layers other than CYTOP. The difference in the high-voltage region among the $I_{T, \text { cross }}(U)$ curves for the three different light wavelengths is attributed to the fact that the maximal phase difference $\Phi_{\max }=2 \pi\left(n_{e}-n_{o}\right) d / \lambda$ depends on the wavelength (for our sample $\Phi_{\max } \approx 2 \pi$ at $\lambda=633$ $\mathrm{nm}, \Phi_{\max } \approx 2.4 \pi$ at $\lambda=532 \mathrm{~nm}$, and $\Phi_{\max } \approx 2.9 \pi$ at $\lambda=450 \mathrm{~nm}$ ).

In the micrographs taken with crossed polarizers, optical vorticity is not observed. However, it can be made visible by use of circular polarizers for the input and output light. When the defects with topological charges of $s=+1$ and $s=-1$ are illuminated by left-handed circularly polarized (LCP) light, the light transmitted through the defects has right-handed circularly polarized (RCP) and LCP components, as shown in Figs. 6(c)-6(f) for the beam patterns filtered by the right- and left-circular polarizers, respectively. The output of opposite handedness (RCP) compared with the input polarization (LCP) corresponds to the vortex mode as evidently observed in a doughnutlike beam profile, while the output with unchanged handedness (LCP) gives a negative doughnut of the residual. Although the doughnutlike rings in Figs. 6(c) and 6(d) prove the presence of the vortex component in the right-handed circularly polarized component, it does not provide any information on its phase structure. Therefore, Mach-Zehnder interferometry is performed by use of a Gaussian reference beam. In Fig. 6(g), a resulting interferogram is displayed with the swirling pattern, indicating the presence of the spirals of the light phase (i.e., the generation of the vortex beam). The number of swirling arms directly gives the topological charge $l$ of the vortex beam generated. In this case, the topological charge obtained is $l= \pm 2$, which is consistent, because it is double the topological charge $(s= \pm 1)$ of the illuminated defect. It can be seen in Fig. 6(h) that a defect with the opposite topological charge $s=-1$ yields a vortex beam with the oppositely spiraling phase as proven by the interferogram of the swirling pattern with the opposite handedness.

Not only the transmission patterns but also the interferograms can be simulated by the Jones-matrix method, by vectorial addition of the electric field vectors, $\mathbf{E}_{o}(x, y)$ and $\mathbf{E}_{e}(x, y)$, of the transmitted and the reference Gaussian waves. Figures 6(i) and 6(j) depict the simulated interference patterns, which exhibit the two spiral arms originating from the vortex component of the light, in good agreement with the experimental data [Fig. 6(g) and 6(h)].

The performance of the vortex generation in the present system is evaluated by the so-called conversion efficiency, $\eta$, defined as the portion of the light intensity converted into the vortex mode:

$$
\eta(U)=\frac{I_{\mathrm{RCP}}(U)}{I_{\mathrm{RCP}}(U)+I_{\mathrm{LCP}}(U)},
$$

where the input light is assumed to be LCP. The transmission intensities $I_{\mathrm{RCP}}$ and $I_{\mathrm{LCP}}$ are measured by use of appropriate circularly polarized filters. The voltage 

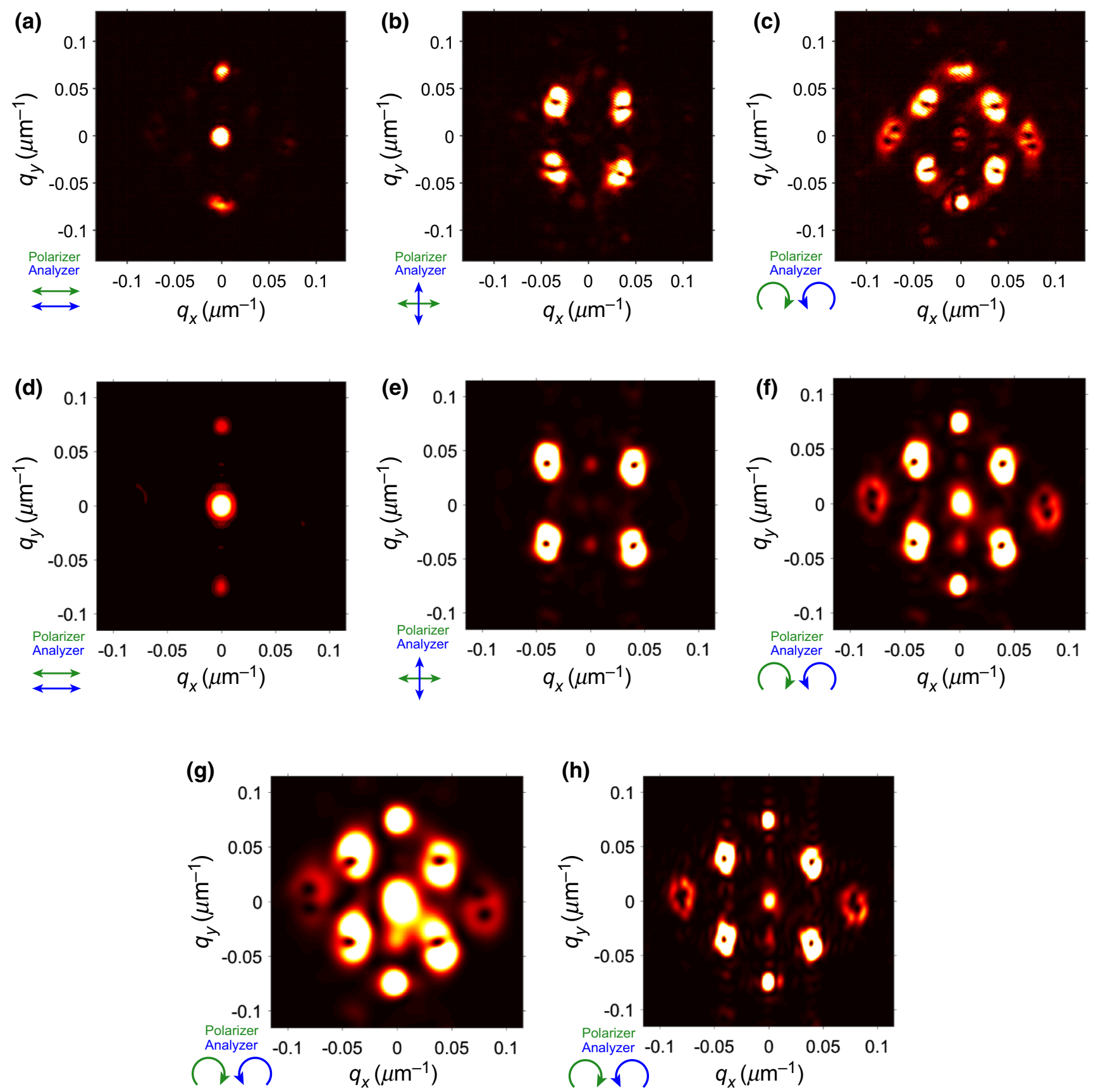

FIG. 9. Diffraction patterns of the defect lattice with a single dislocation at $f=22 \mathrm{~Hz}, U=1.07 U_{\text {th }}$, and $d=26.3 \mu \mathrm{m}$. (a) Between a parallel polarizer and analyzer: the $(0,0),(1,1)$, and $(\overline{1}, \overline{1})$ spots have no vortex structure; the spot intensity is homogeneous. $(\mathrm{b})$ Between a crossed polarizer and analyzer: the $(1,0),(0,1),(0, \overline{1})$ and $(\overline{1}, 0)$ spots exhibit vortex structure; there is no intensity in the middle of the spot. (c) Between a left-circular and a right-circular polarizer: the intensities of the $(1,1)$ and $(\overline{1}, \overline{1})$ spots are homogeneous, the centers of the $(1,0),(0,1),(0, \overline{1})$, and $(\overline{1}, 0)$ spots are dark, and the $(1, \overline{1})$ and $(\overline{1}, 1)$ spots contain two dark regions. Simulated diffraction with use of the birefringence map determined by polarimetry of a dislocation in the grid pattern with $w_{0}=250 \mu \mathrm{m}$ waist radius of the Gaussian illumination (similar to the experimental conditions): (d) between parallel polarizers; (e) between crossed polarizers; (f) between crossed circular polarizers. Dependence on the waist radius $w_{0}$ of illuminating light (between crossed circular polarizers): (g) $w_{0}=150 \mu \mathrm{m}$; (h) $w_{0}=450 \mu \mathrm{m}$.

dependence of $\eta$ is presented in Fig. 7(b). It can be seen that the $\eta(U)$ curves are of the same character as those of $I_{T \text {,cross }}(U)$ shown in Fig. 7(a). $\eta$ takes the highest values at the voltages where the effective phase shift $\Phi$ approaches $\pi$, corresponding to the maxima of $I_{T, \text { cross }}(U)$. This result matches our expectations and agrees with earlier studies that confirmed a similar feature in other systems $[48,52,55]$. 


\section{Vortex-beam generation by diffraction from defect-lattice dislocations}

In the previous sections, we consider the single topological defects and their uniform square lattice. However, imperfectness of the lattice order often leads to the formation of dislocations like in atomic crystals. Thus, the present system actually has another level of homotopically distinct states around these dislocations. The dislocations in the lattice structure may be induced either spontaneously after voltage jumps or intentionally by illumination of the lattice with a focused IR laser [59]. An example of such a dislocation is presented in Figs. 8(a) and 8(b). The director mapping is performed by polarimetry, as shown in Figs. 8(c) and 8(d). The result clearly shows the dislocation possessing a Burgers vector parallel to the $x$ axis.

The presence of the dislocation breaks the fourfold symmetry of the square defect lattice; that is, the directions parallel $(x)$ to and perpendicular $(y)$ to the Burgers vector are no longer equivalent. This symmetry breaking modifies the diffraction pattern. Figures 9(a)-9(c) show the measured diffraction pattern of the defect lattice with a single dislocation located in the center of the illuminated area. The pattern changes with the polarization state as shown in Fig. 9(a) between a parallel polarizer and analyzer $(\beta=0)$, in Fig. 9(b) between a crossed polarizer and analyzer $(\beta=$ 0 ), and in Fig. 9(c) between a left-circular and a rightcircular polarizer. It is clearly seen that the central beam and the $(1,1)$ and $(\overline{1}, \overline{1})$ spots exhibit no vortex character. In contrast, the centers of the $(1,0),(0,1),(\overline{1}, 0)$, and $(0, \overline{1})$ spots are dark, implying that these diffracted beams possess a vortex character. Moreover, the $(1, \overline{1})$ and $(\overline{1}, 1)$ spots exhibit two dark regions indicating optical singularities.

To calculate the diffracted light on the grid pattern with a dislocation, we take the projection of the director structure determined by polarimetry (seen in Fig. 8). By having the spatial distribution of the magnitude and azimuthal
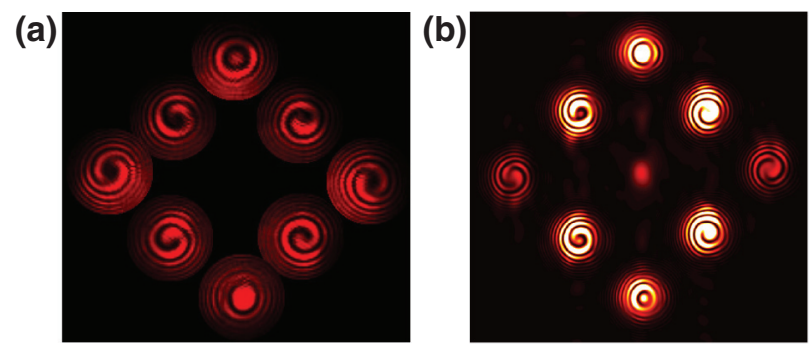

FIG. 10. (a) A montage of the experimental interference patterns of a Gaussian reference beam with the diffraction spots resulting from a sample in between a left-circular and a rightcircular polarizer. For $(1,1)$ and $(\overline{1}, \overline{1})$ the pattern is circular $(l=0)$. For $(1,0),(0,1),(0, \overline{1})$, and $(\overline{1}, 0)$ the spiral has one arm $(l=+1$ or -1$)$. For $(1, \overline{1})$ and $(\overline{1}, 1)$ the spiral has two arms $(l=+2$ or -2$)$. (b) Interference patterns of the simulated diffraction with eight Gaussian reference beams showing good agreement with (a). angle of the effective birefringence, we can position the center of illuminating light with a Gaussian profile in the core of the dislocation. Using the Jones-matrix method, we calculate the distribution of output electric fields and the diffraction similarly to the dislocation-free case described in Sec. III A. The calculated diffraction patterns corresponding to the different polarization states shown in Figs. 9(a), 9(b), and 9(c) can be seen in Figs. 9(d), 9(e), and 9(f), respectively. In all cases here, the waist radius $w_{0}=250 \mu \mathrm{m}$ is used, which is similar to the experimental conditions. For $(1, \overline{1})$ and $(\overline{1}, 1)$, the most spectacular in Fig. 9(f), we obtain the two dark regions found also in the experiments.

We suspect that the presence of two dark spots in $(1, \overline{1})$ and $(\overline{1}, 1)$ may be the result of the limited area of illumination. To investigate this hypothesis, we calculate the diffraction patterns as a function of the waist radius $w_{0}$ of the Gaussian input beam. The dependence of the diffraction pattern (with use of crossed circular polarizers) on the waist radius can be seen in Figs. 9(g) and 9(h) corresponding to $w_{0}=150 \mu \mathrm{m}$ and $w_{0}=450 \mu \mathrm{m}$, respectively. For the smaller waist seen in Fig. 9(g), the presence of the two dark spots in $(1, \overline{1})$ and $(\overline{1}, 1)$ is more pronounced, while for the larger beam size [Fig. 9(h)], the two dark regions are merged, showing single doughnuts, as expected. Because of the limited field of view available for the polarimetry, the effect of a significantly larger beam size cannot be studied. (In Fig. 8, only a fraction of the entire field of view is plotted for better visibility.) The calculated diffraction pattern in Fig. 9(h) matches the experimental finding [Fig. 9(c)] because of the approximate matching of the illuminating-beam sizes.

To experimentally confirm the topological states of the diffracted beams, a Gaussian reference is directed to each diffraction spot with use of a left-handed and a righthanded circular polarizer for input and output, respectively. The montage in Fig. 10(a) summarizes the behavior of the indexed diffraction spots (except for $(0,0)$ ). The interference patterns for the $(1,0),(0,1),(0, \overline{1})$, and $(\overline{1}, 0)$ spots are spirals with one arm; the spirals rotate counterclockwise for $(1,0)$ and $(0, \overline{1})$ but clockwise for $(\overline{1}, 0)$ and $(0,1)$. They correspond to optical vortices with a topological charge of $l= \pm 1$. On the other hand, the interference patterns of $(1,1)$ and $(\overline{1}, \overline{1})$, located along the direction normal to the Burgers vector, are circular (i.e., they are Gaussian beams). The remaining spots, $(1, \overline{1})$ and $(\overline{1}, 1)$, located along the direction of the Burgers vector, exhibit a spiral interference pattern with two arms rotating clockwise and counterclockwise, respectively. They correspond to optical vortices with a topological charge of $|l|=2$.

For justification of the experimental findings, we calculate the interference of the simulated diffraction spots with eight Gaussian reference beams and present them in Fig. 10(b). In all cases, the simulated interference reproduces the experimentally obtained spirals with all 

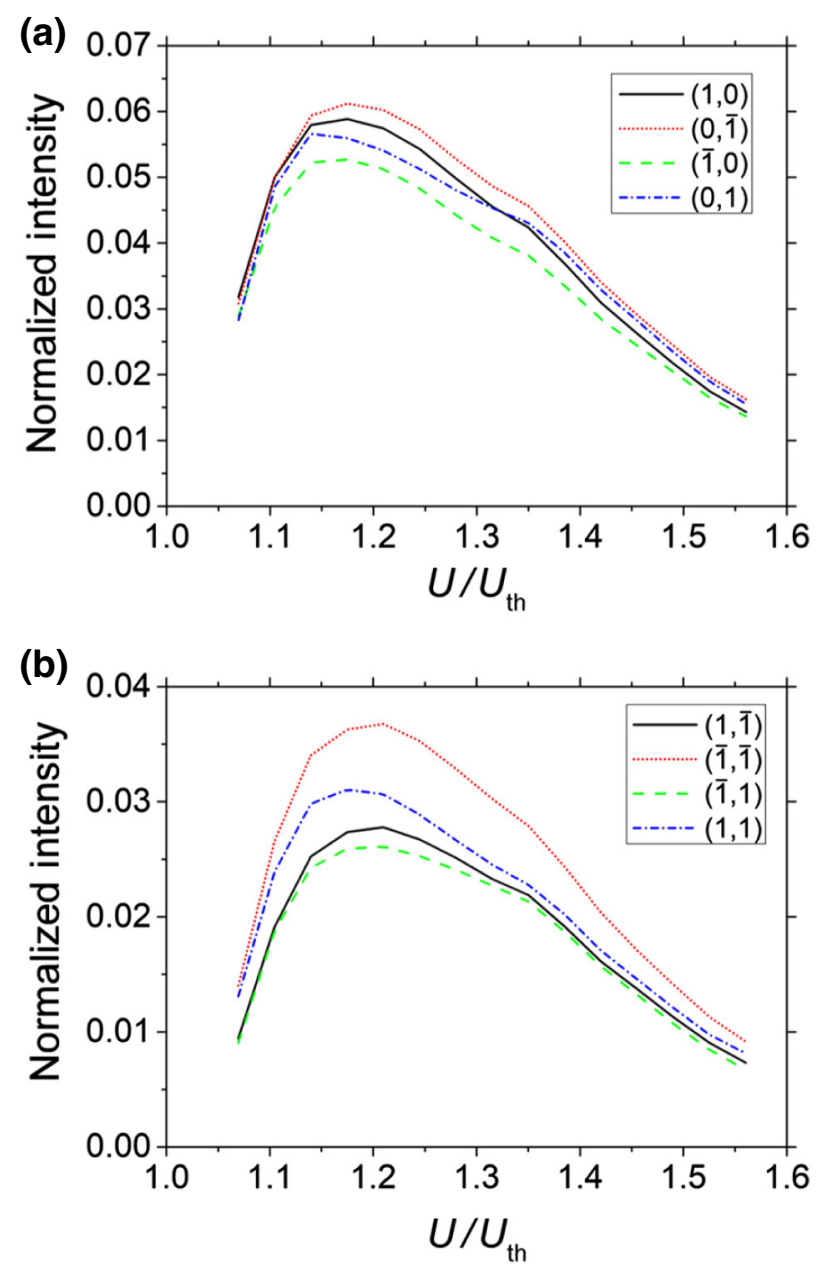

FIG. 11. Voltage dependence of the diffraction efficiency of diffraction spots for a dislocation in the defect lattice for a sample placed in between left-handed and right-handed circular polarizers (a) for $(1,0),(0,1),(0, \overline{1})$, and $(\overline{1}, 0)$ spots and (b) for $(1, \overline{1})$ and $(\overline{1}, 1)$ spots.

important characteristics, including the number of arms and helicities.

The relative intensity of the diffraction spots (i.e., the diffraction efficiency) depends on the applied voltage. Figure 11 depicts the relative intensities of spots belonging to $l= \pm 1$ [Fig. 11(a)] and $l= \pm 2$ [Fig. 11(b)] obtained for a sample placed between a left-handed and a right-handed circular polarizer with the same method as before. The largest diffraction efficiencies are obtained for the voltage where the retardation is $\Phi \approx \pi$, similarly to the vortexbeam generation from the single defects. At the same voltage, the intensity of the direct $(0,0)$ beam transmitted by two left-circular polarizers decreases to zero (i.e., under such conditions all the light goes into the diffracted beams).

\section{CONCLUSION}

We show that lattices of the topological defects appearing during the recently discovered inhomogeneous director reorientation of homeotropic layers of nematic liquid crystals with negative dielectric anisotropy are convenient targets for studying the generation of optical vortices. As demonstrated, vortex generation may occur at two levels (i.e., at two length scales). At the first level, each individual defect converts part of the circularly polarized light into a vortex beam with opposite circular polarization, as is clearly proven by microscopy. Beams diffracted on a regular defect lattice, however, do not carry vorticity; this is attributed to the fact that the diameter of the laser beam used for diffraction much exceeds the length scale of the deformation around a defect. At the second level, a dislocation of the lattice structure represents a topological defect at another, larger length scale, which can be of the same order as the diameter of the laser beam. Then diffraction on a single dislocation is possible, which results in diffracted beams with an optical vortex character. The topological charge of these optical vortices depends on the Burgers vector as well as on the order (the Miller indices) of the diffraction.

The vortex generation and the diffraction efficiencies depend strongly on the applied voltage. Efficiencies are maximal when the retardation of the nematic layer equals $\Phi=\pi$. For diffraction on a dislocation, all light is converted into the diffracted (mostly vorticity-carrying) beams.

As regular defect lattices as well as dislocations in them can be fabricated reliably and reproducibly, the effect studied has the potential of practical application as a source of optical vortex beams. For actual device preparation, fixing the positions of the defects and their dislocations may be a crucial point. Partial polymerization of the distorted structure [60] is a tool to reach that goal. In the case when the optical vortex generation is done by varying the wavelength, or electronic switching on and off is needed, the system presented here may offer a better alternative to the currently used techniques of finite-resolution spatial light modulators or vortex retarder half wave plates made for a specific wavelength.

\section{ACKNOWLEDGMENTS}

This work was supported by the Hungarian National Research, Development, and Innovation Office (Grants No. PD 121019 and No. FK 125134) and the Japan Society for the Promotion of Science-Hungarian Academy of Sciences bilateral project (Grant No. NKM-49/2016).

[1] L. Allen, Stephen M. Barnett, and Miles J. Padgett, Optical Angular Momentum (IOP Publishing, Bristol and Philadelphia, 2003).

[2] M. Soskin, S. V. Boriskina, Y. Chong, M. R. Dennis, and A. Desyatnikov, Singular optics and topological photonics, J. Opt. 19, 010401 (2017). 
[3] M. Reuss, J. Engelhardt, and S. W. Hell, Birefringent device converts a standard scanning microscope into a STED microscope that also maps molecular orientation, Opt. Expr. 18, 1049 (2010).

[4] P. C. Maurer, J. R. Maze, P. L. Stanwix, L. Jiang, A. V. Gorshkov, A. A. Zibrov, B. Harke, J. S. Hodges, A. S. Zibrov, A. Yacoby, D. Twitchen, S. W. Hell, R. L. Walsworth, and M. D. Lukin, Far-field optical imaging and manipulation of individual spins with nanoscale resolution, Nat. Phys. 6, 912 (2010).

[5] A. T. O’Neil, I. MacVicar, L. Allen, and M. J. Padgett, Intrinsic and Extrinsic Nature of the Orbital Angular Momentum of a Light Beam, Phys. Rev. Lett. 88, 053601 (2002).

[6] J. E. Curtis and D. G. Grier, Structure of Optical Vortices, Phys. Rev. Lett. 90, 133901 (2003).

[7] A. S. Desyatnikov, V. G. Shvedov, A. V. Rode, W. Krolikowski, and Y. S. Kivshar, Photophoretic manipulation of absorbing aerosol particles with vortex beams: Theory versus experiment, Opt. Expr. 17, 8201 (2009).

[8] V. G. Shvedov, A. S. Desyatnikov, A. V. Rode, Y. V. Izdebskaya, W. Z. Krolikowski, and Y. S. Kivshar, Optical vortex beams for trapping and transport of particles in air, Appl. Phys. A 100, 327 (2010).

[9] Y. Yan, G. Xie, M. P. J. Lavery, H. Huang, N. Ahmed, C. Bao, Y. Ren, Y. Cao, L. Li, Z. Zhao, A. F. Molisch, M. Tur, M. J. Padgett, and A. E. Willner, High-capacity millimetrewave communications with orbital angular momentum multiplexing, Nat. Commun. 5, 4876 (2014).

[10] S. R. Nersisyan, N. V. Tabiryan, D. Mawet, and E. Serabyn, Improving vector vortex waveplates for high-contrast coronagraphy, Opt. Expr. 21, 8205 (2013).

[11] A. Aleksanyan and E. Brasselet, Vortex coronagraphy from self-engineered liquid crystal spin-orbit masks, Opt Lett. 41, 5234 (2016).

[12] A. Aleksanyan, N. Kravets, and E. Brasselet, MultipleStar System Adaptive Vortex Coronagraphy Using a Liquid Crystal Light Valve, Phys. Rev. Lett. 118, 203902 (2017).

[13] V. Yu. Bazenhof, M. V. Vasnetsov, and M. S. Soskin, Laser beams with screw dislocation in their wavefronts, JETP Lett. 52, 429 (1990) [Pis'ma Zh. Eksp. Teor. Fiz. 52, 1037-1039 (1990)].

[14] D. G. Grier, A revolution in optical manipulation, Nature 424, 810 (2003).

[15] S. Nersisyan, N. Tabiryan, D. M. Steeves, and B. R. Kimball, Fabrication of liquid crystal polymer axial waveplates for UV-IR wavelengths, Opt. Expr. 17, 11926 (2009).

[16] P. G. de Gennes and J. Prost, The Physics of Liquid Crystals (Clarendon Press, Oxford, 1993).

[17] M. Humar, M. Skarabot, M. Ravnik, S. Zumer, I. Poberaj, D. Babic, and I. Musevic, Electrically tunable diffraction of light from 2D nematic colloidal crystals, Eur. Phys. J. E 27, 73 (2008).

[18] M. Y. Xu, M. J. Zhou, Y. Xiang, P. Salamon, N. Eber, and A. Buka, Domain structures as optical gratings controlled by electric field in a bent-core nematic, Opt. Express 23, 15224 (2015).

[19] Y. Xiang, H-Z Jing, Z-D. Zhang, W-J. Ye, M-Y Xu, E. Wang, P. Salamon, N. Eber, and A. Buka, Tunable Optical Grating based on the Flexoelectric Effect in a Bent-core
Nematic Liquid Crystal, Phys. Rev. Applied 7, 064032 (2017).

[20] I. Musevic, M. Skarabot, U. Tkalec, M. Ravnik, and S. Zumer, Two-dimensional nematic colloidal crystals selfassembled by topological defects, Science 313, 954 (2006).

[21] N. Eber, P. Salamon, and A. Buka, Electrically induced patterns in nematics and how to avoid them, Liq. Cryst. Rev. 4, 101 (2016).

[22] Y. Geng, J. H. Noh, I. Drevensek-Olenik, R. Rupp, G. Lenzini, and J. P. F. Lagerwall, High-fidelity spherical cholesteric liquid crystal Bragg reflectors generating unclonable patterns for secure authentication, Sci. Rep. 6, 26840 (2016).

[23] M. Schwartz, G. Lenzini, Y. Geng, P. B. Ronne, P. Y. A. Ryan, and J. P. F. Lagerwall, Cholesteric liquid crystal shells as enabling material for information-rich design and architecture, Adv. Mater. 30, 1707382 (2018).

[24] T. H. Ware, M. E. McConney, J. J. Wie, V. P. Tondiglia, and T. J. White, Voxelated liquid crystal elastomers, Science 347, 982 (2015).

[25] T. J. White and D. J. Broer, Programmable and adaptive mechanics with liquid crystal polymer networks and elastomers, Nat. Mater. 14, 1087 (2015).

[26] G. Babakhanova, T. Turiv, Y. Guo, M. Hendrikx, Q-H. Wei, A. P. H. J. Schenning, D. J. Broer, and O. D. Lavrentovich, Liquid crystal elastomer coatings with programmed response of surface profile, Nat. Commun. 9, 456 (2018).

[27] L. Marrucci, C. Manzo, and D. Paparo, Optical Spinto-Orbital Angular Momentum Conversion in Inhomogeneous Anisotropic Media, Phys. Rev. Lett. 96, 163905 (2006).

[28] L. Marrucci, E. Karimi, S. Slussarenko, B. Piccirillo, E. Santamato, E. Nagali, and F. Sciarrino, Spin-to-orbital conversion of the angular momentum of light and its classical and quantum applications, J. Opt. 13, 064001 (2011).

[29] S. C. McEldowney, D. M. Shemo, R. A. Chipman, and P. K. Smith, Creating vortex retarders using photoaligned liquid crystal polymers, Opt. Lett. 33, 134 (2008).

[30] S. C. McEldowney, D. M. Shemo, and R. A. Chipman, Vortex retarders produced from photo-aligned liquid crystal polymers, Opt. Expr. 16, 7295 (2008).

[31] S. Slussarenko, A. Murauski, T. Du, V. Chigrinov, L. Marrucci, and E. Santamato, Tunable liquid crystal q-plates with arbitrary topological charge, Opt. Expr. 19, 4085 (2011).

[32] P. Chen, B.-Y. Wei, W. Ji, S.-J. Ge, W. Hu, F. Xu, V. Chigrinov, and Y.-Q. Lu, Arbitrary and reconfigurable optical vortex generation: A high-efficiency technique using director-varying liquid crystal fork gratings, Photon. Res. 3, 133 (2013).

[33] P. Chen, W. Ji, B.-Y. Wei, W. Hu, V. Chigrinov, and Y.-Q. $\mathrm{Lu}$, Generation of arbitrary vector beams with liquid crystal polarization converters and vector-photoaligned q-plates, Appl. Phys. Lett. 107, 241102 (2015).

[34] P. Chen, Y.-Q. Lu, and W. Hu, Beam shaping via photopatterned liquid crystals, Liq. Cryst. 43, 2051 (2016).

[35] P. Chen, S.-J. Ge, L.-L. Ma, W. Hu, V. Chigrinov, and Y.-Q. Lu, Generation of Equal-Energy Orbital Angular Momentum Beams via Photopatterned Liquid Crystals, Phys. Rev. Appl. 5, 044009 (2016). 
[36] P. Chen, S.-J. Ge, W. Duan, B.-Y. Wei, G.-X. Cui, W. $\mathrm{Hu}$, and Y.-Q. Lu, Digitalized geometric phases for parallel optical spin and orbital angular momentum encoding, ACS Photonics 4, 1333 (2017).

[37] P. Chen, L.-L. Ma, W. Duan, J. Chen, S.-J. Ge, Z.-H. Zhu, M.-J. Tang, R. Xu, W. Gao, T. Li, W. Hu, and Y.-Q. Lu, Digitalizing self-assembled chiral superstructures for optical vortex processing, Adv. Mater. 30, 1705865 (2018).

[38] Z. Ji, X. Zhang, Y. Zhang, Z. Wang, I. Drevensek-Olenik, R. Rupp, W. Li, Q. Wu, and J. Xu, Electrically tunable generation of vectorial vortex beams with micro-patterned liquid crystal structures, Chin. Opt. Lett. 15, 070501 (2017).

[39] L. K. Migara and J.-K. Song, Standing wave-mediated molecular reorientation and spontaneous formation of tunable, concentric defect arrays in liquid crystal cells, NPG Asia Mater. 10, e459 (2018).

[40] B. Son, S. Kim, Y. H. Kim, K. Käläntär, H.-M. Kim, H.S. Jeong, S. Q. Choi, J. Shin, H.-T. Jung, and Y.-H. Lee, Optical vortex arrays from smectic liquid crystals, Opt. Expr. 22, 4699 (2014).

[41] P. J. Ackerman, Z. Qi, Y. Lin, C. W. Twombly, M. J. Laviada, Y. Lansac, and I. I. Smalyukh, Laser-directed hierarchical assembly of liquid crystal defects and control of optical phase singularities, Sci. Rep. 2, 414 (2012).

[42] P. J. Ackerman, Z. Qi, and I. I. Smalyukh, Optical generation of crystalline, quasicrystalline, and arbitrary arrays of torons in confined cholesteric liquid crystals for patterning of optical vortices in laser beams, Phy. Rev. E 86, 021703 (2012).

[43] D. Voloschenko and O. D. Lavrentovich, Optical vortices generated by dislocations in a cholesteric liquid crystal, Opt. Lett. 25, 317 (2000).

[44] H. Choi, J. H. Woo, J. W. Wu, D.-W. Kim, T.-K. Lim, and S. H. Song, Holographic inscription of helical wavefronts in a liquid crystal polarization grating, Appl. Phys. Lett. 91, 141112 (2007).

[45] B.-y. Wei, W. Hu, Y. Ming, F. Xu, S. Rubin, J.-g. Wang, V. Chigrinov, and Y.-q. Lu, Generating switchable and reconfigurable optical vortices via photopatterning of liquid crystals, Adv. Mater. 26, 1590 (2014).

[46] Y. Ma, B. Y. Wei, L. Y. Shi, A. K. Srivastava, V. G. Chigrinov, H-S. Kwok, W. Hu, and Y. Q. Lu, Fork gratings based on ferroelectric liquid crystals, Opt. Expr. 24, 5822 (2016).

[47] E. Brasselet, N. Murazawa, H. Misawa, and S. Juodkazis, Optical Vortices from Liquid Crystal Droplets, Phys. Rev. Lett. 103, 103903 (2009).

[48] E. Brasselet and C. Loussert, Electrically controlled topological defects in liquid crystals as tunable spin-orbit encoders for photons, Opt. Lett. 36, 719 (2011).

[49] E. Brasselet, Tunable Optical Vortex Arrays from a Single Nematic Topological Defect, Phys. Rev. Lett. 108, 087801 (2012)

[50] D. Ganic, X. Gan, M. Gu, M. Hain, S. Somalingam, S. Stankovic, and T. Tschudi, Generation of doughnut laser beams by use of a liquid-crystal cell with a conversion efficiency near 100\%, Opt. Lett. 27, 1351 (2002).

[51] R. Barboza, U. Bortolozzo, G. Assanto, E. VidalHenriquez, M. G. Clerc, and S. Residori, Harnessing Optical Vortex Lattices in Nematic Liquid Crystals, Phys. Rev. Lett. 111, 093902 (2013).
[52] C. Loussert, K. Kushnir, and E. Brassele, Q-plates microarrays for parallel processing of the photon orbital angular momentum, Appl. Phys. Lett. 105, 121108 (2014).

[53] Q. Wang, X. W. Sun, and P. Shum, Generating doughnutshaped beams with large charge numbers by use of liquidcrystal spiral phase plates, Appl. Opt. 43, 2292 (2004).

[54] Q. Wang, X. W. Sun, P. Shum, and X. J. Yin, Dynamic switching of optical vortices with dynamic gammacorrection liquid crystal spiral phase plate, Opt. Expr. 13, 10285 (2005).

[55] C. Loussert, U. Delabre, and E. Brasselet, Manipulating the Orbital Angular Momentum of Light at the Micron Scale with Nematic Disclinations in a Liquid Crystal Film, Phys. Rev. Lett. 111, 037802 (2013).

[56] M. E. McConney, A. Martinez, V. P. Tondiglia, K. M. Lee, D. Langley, I. I. Smalyukh, and T. J. White, Topography from topology: Photoinduced surface features generated in liquid crystal polymer networks, Adv. Mater. 25, 5880 (2013).

[57] H. Yoshida, K. Asakura, J. Fukuda, and M. Ozaki, Threedimensional positioning and control of colloidal objects utilizing engineered liquid crystalline defect networks, Nat. Commun. 6, 7180 (2015).

[58] Y. V. Izdebskaya, A. S. Desyatnikov, G. Assanto, and Y. S. Kivshar, Dipole azimuthons and vortex charge flipping in nematic liquid crystals, Opt. Expr. 19, 21457 (2011).

[59] Y. Sasaki, V. S. R. Jampani, C. Tanaka, N. Sakurai, S. Sakane, K. V. Le, F. Araoka, and H. Orihara, Large-scale self-organization of reconfigurable topological defect networks in nematic liquid crystals, Nature Commun. 7, 13238 (2016).

[60] Y. Sasaki, M. Ueda, K. V. Le, R. Amano, S. Sakane, S. Fujii, F. Araoka, and H. Orihara, Polymer-stabilized micropixelated liquid crystals with tunable optical properties fabricated by double templating, Adv. Mater. 29, 1703054 (2017).

[61] S. Aya, Y. Sasaki, H. Takezoe, K. Ishikawa, K. Ema, T. Hikima, M. Takata, and F. Araoka, Thermodynamically anchoring-frustrated surface to trigger bulk discontinuous orientational transition, Langmuir 32, 10545 (2016).

[62] M. Shribak and R. Oldenbourg, Sensitive measurements of two-dimensional birefringence distributions using nearcircularly polarized beam, Proc. SPIE 4819, 56 (2002).

[63] A. Rapini, Umbilics: Static properties and shear-induced displacements, J. Phys. (France) 34, 629 (1973).

[64] I. Dierking, M. Ravnik, E. Lark, J. Healey, G. P. Alexander, and J. M. Yeomans, Anisotropy in the annihilation dynamics of umbilic defects in nematic liquid crystals, Phys. Rev. E 85, 021703 (2012).

[65] I. Dierking and P. Archer, Imaging liquid crystal defects, RSC Adv. 3, 26433 (2013).

[66] E. Hecht, Optics (Addison Wesley, San Francisco, 2002), 4th ed.

[67] U. Mur, S. Copar, G. Posnjak, I. Musevic, M. Ravnik, and S. Zumer, Ray optics simulations of polarised microscopy textures in chiral nematic droplets, Liq. Cryst. 44, 679 (2017).

Correction: The email address was missing for an additional corresponding author at publication and has now been inserted. 